\title{
Virtual Team Performance Depends on Distributed Leadership
}

\author{
Nico van Dijk and Joost Broekens \\ Media Technology MSc, Leiden University \\ Man-Machine Interaction Group, Delft University of Technology \\ nicovandijk@gmail.com, joost.broekens@gmail.com
}

\begin{abstract}
In this paper we present a detailed analysis of World of Warcraft virtual team collaboration. A number of competitive synchronous virtual teams were investigated in-situ and unobtrusively. We observed a large gap in team performance between the various teams. An initial statistic study showed that, in teams of this level, individual player performance was not the primary driver for the large discrepancy in team performance. This led to the argument that differences in intra-team collaboration and communication might be a significant driver for the discrepancy in team performance. In total 16 hours of audio recordings of gaming sessions of virtual teams were analyzed. The analysis indicates that distributed leadership instead of authoritative leadership is more common in successful synchronous virtual teams.
\end{abstract}

Keywords: Virtual Teams, Collaboration, Communication, Distributed Leadership, MMORPG.

\section{Introduction}

Recently, the use of computer games for research purposes has become increasingly popular [1]. Often this research is focused on researching how games can be made to be more entertaining or educational, but also on how video games can be used as research tools to study more general phenomenon (see, e.g., [2,3]). Topics such as team strategy and decision making are being researched in a video game context [4].

Virtual teams have become more prevalent in various fields of business and research as Computer Mediated Communication (CMC) has become more widespread and accessible. A recent survey by the Gartner group found that more than $60 \%$ of professional employees work in virtual teams $[5,6,7]$. It is expected that the business world will increasingly use CMC [6]. Additionally, a significant amount of study on virtual teams and team training in virtual worlds is being done in defense and disaster relief related areas $[8,9]$. Thus it is very valuable to investigate the internal communication of teams already operating under these conditions.

There are still gaps in knowledge about virtual team collaboration and communication, in particular when it comes to studying virtual teams in-situ. Therefore it is worthwhile to study teams with a real-time, unobtrusive method that can be practically implemented $[10,11]$. 
In online computer games, especially Massively Multiplayer Online games (MMO), $\mathrm{CMC}$ is the rule rather than the exception and this creates interesting opportunities to study Virtual Teams in-situ. MMO's serve as particularly good environments for this type of research for a number of reasons. MMO's have active functioning teams that share a common task and goal. Due to the amount of players there is a large diversity of teams available to collect data on. The members of these teams are self-motivated to participate in their shared activity. Members can be observed unobtrusively and in realtime while being engaged in sessions of collaborative problem solving. Players invest quite some effort in the game and their team indicating that the team is indeed viewed by the players as an online team with a common underlying interest. Finally, player behavior with respect to leadership and other higher level organizational skills is quite consistent with respect to the player's real-world behavior (see e.g. [12]). We studied communication and organization behavior in synchronous virtual teams in the popular MMO World of Warcraft (WoW).

We set out to investigate the relation between team performance and team structure. More precisely, we wanted to know if there was a relation between the quality of the team and the type of collaboration the team used. The study setup was unique in that the participating teams operated in duplicate environments, while still being insitu and unobtrusive. This means that variation in team behavior is caused by the players and their interactions. The teams were unaware of the study, providing an excellent opportunity to study the teams in the wild. The data was analyzed qualitatively. This paper is organized as follows. In section 2 the relation between virtual teams and MMO's is explained. In section 3 our study setup is explained. In section 4 the results are presented. Section 5 and 6 present the discussion and conclusion respectively.

\section{Virtual Teams and MMO's}

Cohen and Baily define a team as "a collection of individuals who are interdependent in their tasks, who share responsibility for outcomes, who see themselves and who are seen by others as an intact social entity embedded in one or more larger social systems, and who manage their relationship across organizational boundaries."[13] This definition is general enough to capture traditional, as well as virtual teams while precisely identifying the defining features of a team: its unity of purpose, its identity as a social structure, and its members' shared responsibility for outcomes.

Rosen et. al define a virtual team as a "group of geographically, organizationally and/or time dispersed workers brought together by information and telecommunication technologies to accomplish one or more organizational tasks” [14, 15, 16].

\subsection{Virtual Teams in World of Warcraft}

The Virtual teams that were studied in WoW adhere to the requirements of both of these definitions. Players form large collectives called guilds. From these guilds smaller teams of players are formed into what is known as a 25-player raid group. We consider a 25-player raid group as a team (please note that as all guilds in our study had one 25-player raid group, the terms guild, team and 25-player raid group will be 
used interchangeable and all refer to the guild's 25-player raid group). This 25-player raid group attempts to succeed in the bigger challenges that can be found inside the game environment. The members of these groups are interdependent in their tasks, communicate only through computer mediated means and are driven to succeed in collectively overcoming the challenges that the game offers. Considering the time investment that is needed from the team to defeat these in-game challenges (typically more than 20 hours of play time) and the amount of communication occurring we assume that these challenges are complex problems. While their livelihood does not depend on their success in the game the amount of time team members dedicate to their team is significant enough that we can consider the team to generally be very well motivated to succeed. There are many similarities between our virtual teams and the problem characteristics of virtual teams described by the Macro-cognitive Model of Team Collaboration [17]. Virtual teams in WoW operate under time pressure and often not all information or knowledge about a challenge is available for players. There usually is a large amount of knowledge to handle and the interface with the game is complex. Player's often custom design their user interface to facilitate their functioning in the team. The teams are distributed, culturally diverse, usually employ a fairly strict--although not very deep-hierarchy, and their members have unique roles and regularly rotate within the team. The operational tasks are also similar, team decisions about the planned tactics have to be made, the players have to develop a shared understanding of the situation and transform available information into a functional tactic that works for the team in the field. One difference is that the teams tend to work synchronously instead of asynchronous. Asynchronous communication usually only occurs during the planning phase for content when the teams use forums to exchange information.

\subsection{Distributed and Authoritative Leadership}

The following two descriptions for collaboration styles were based on phenomenon found in the data initially. When a team employs authoritative leadership, there is a designated person that functions as leader. Communication is characterized by giving instructions, explaining decisions and asking about status information (i.e., information important for decisions, planning and action selection is typically gathered and analyzed by the leader). The leader decides what the team does.

In contrast, in distributed leadership, leading never rests with just a single person but rather leadership is a role often and quickly passed between different members of a team. Teams that use distributed leadership are characterized by high communicative interaction between its various members. Focus is shifted away from the individual actions of leaders and their personal qualities. Instead the practice of leadership as "interactions between the leaders, followers and situation" becomes more important, and this interaction is used to plan, decide and act [8].

\section{Research Design}

We now explain our research design used to investigate the relation between team performance and team structure. The chosen gaming platform enabled us to study intra-team communication in great detail: all data can be logged, and the researcher's 
real motives could be hidden. These two aspects have not often been exploited in similar studies. Given the large amount and variability of data collected, and the relatively open-endedness of a participatory field study, a qualitative study design was more suitable as compared to a quantitative hypothesis-focused approach. This allowed our data analysis to be more explorative.

It is plausible to assume that differences in team performance are explained by individual player performance (i.e., better players in better teams). We carefully checked this assumption before starting the detailed and time consuming task of monitoring and qualitatively analyzing teams in order to find effects of team collaboration on performance. We polled a random set of 150 players in the top 4000 guilds in Europe (top 4000 selected according to www.wowprogress.com, 5 players per guild, 30 guilds in total). These players were ranked according to a measure for player quality based on several game-performance elements extracted from the website europe.wowarmory.com (official website of the developer Blizzard containing actual game data). These elements included: number of bosses killed in 5 player dungeons, number of 5 player dungeons entered, number of bosses killed in 10 player dungeons, number of 10 player instances entered, number of Quests completed, number of Daily Quests completed, date of reaching level 80.

In essence, all elements define a player's activity in the game, except the last which defines how "senior" a player is with respect to how long the player plays the game. We excluded the activity related to the team performance we will be observing (i.e. the 25-raid group activities). Players with high activity and seniority have more experience and are assumed to be better performing.

To investigate if player rank relates to team rank in the top 4000 teams, we statistically analyzed this relation. This showed a significant correlation between guild rank and player rank among the top 4000 teams $(n=150, r=-0.273$. $p=0.001)$. However, when considering only the teams ranked 1000 to 4000 , there was no significant correlation ( $\mathrm{n}=99, \mathrm{r}=-0.127, \mathrm{p}=\mathrm{ns})$. This indicates that within the well-performing set of ranked teams, not belonging to the absolute top 1000 , individual player quality is not related to team performance. Thus, in the top 1000 to 4000 other factors, such as collaboration and communication explain the difference in team rank.

Based on these results we decided to embed an avatar (representation of the researcher in the game) in European guilds ranked between position 1000 and 4000. These avatars would be active in the guild's 25 -raid group for several weeks / months. The main goal for our study design was to obtain all our data unobtrusively and insitu. As such, the fact that research was being done was hidden to the virtual team. In ethnographic fieldwork it is quite accepted to study subjects without formally requesting their consent. We consider our method of acquiring data to share many similarities with this process.

A secondary goal was to gather data on teams during encounters they had not defeated before. Prior experience by the first author indicated that a team's communication pattern changes drastically when teams have defeated the same challenge several times. To eliminate this factor only data recorded during moments teams were engaging new content was analyzed.

Four teams to gather data from were selected based on guild rank (according to www.wowprogress.com and our previous analysis), and if the guild had already finished a newly published challenge (teams attempted similar new content). As clarified in 2.1 we use the term guild and team interchangeably and they refer to the same entity. 
- Team A - position $\sim 1000$

- Team B - position $\sim 1300-1500$

- Team C - position $\sim 1500-1800$

- Team D - position $\sim 4000$

The differences in performance between guilds ranked near position 1000 and guilds ranked near position 4000 are significant. For example, Guild 'Going Postal' ranked EU 1025 achieved the 'Heroic Lord Jaraxxus' challenge on October 3rd 2009, while Guild 'Ypsilon' ranked EU 3747 achieved the same challenge on December $21^{\text {st }}$ 2009. This represents a difference of more than two months, between guilds that both play on 3 scheduled evenings each week. The boss became accessible to both teams in the first week of September 2009, meaning 'Going Postal' was close to $400 \%$ faster, given that they played on similar schedules. This is a major difference given the fact that these guilds are ranked and being the best team and achieving a higher rank on the server or continent is an important status achievement in WoW.

Data was gathered in several different ways. WoW raid groups use Voice Over Internet Protocol (VOIP) applications during their team sessions to facilitate their intra-team communication (e.g. Teamspeak or Ventrilo). These voice communications were recorded. Audio recordings were started the moment a group was fully gathered and players got on their way to the respective challenge they would attempt that evening. Audio recordings were concluded when the group was disbanded at the end of the evening.

\subsection{Data Analysis}

Most interesting were the VOIP audio recordings, these seemed to contain the highest frequency of communication. Nivo8 was used to structure the data. The data analysis was explorative and a coding scheme was developed to disseminate the communication. After initial scanning, it seemed necessary to develop a more structured and generalized coding scheme that could be applied to both the audio recordings and the chat logs as well as be generic enough to be able to be applied to virtual teams in different environments.

All coding of the audio files was done manually. This was a very time intensive process. In total 16 hours of audio data (out of the total 70+ hours recorded) was analyzed. Session length was between 50 minutes and 4 hours long. Every session was coded and annotated down to the second. Six Sessions in total were analyzed, two for Team A, two for Team D, one for Team B and one for Team C. The number of references being created per session varied between approximately 700 and 2800 depending on the length of the session.

To code what happened in the audio and chat, we used two coding schemes. These schemes were able to cover all information exchange within the teams that was related to the challenge they were attempting to succeed in.

- Members: (Officer1, Officer2, Officer3, Officer4, Officer5, Raiders)

- Tactical information: (Instruction, Negotiation, Reflection, Status, Action)

The member scheme represents who speaks. All teams that were studied had defined officers and the remaining members of the team were called raiders. It's worthwhile 
to mention that these guilds determine and assign their own officers inside the game. They are regular players, but in the guild have extra privileges and control over who is member in the guild, as well as carry extra tasks and responsibilities (administration, recruiting, conflict management etc.). This self-regulated hierarchy was used to assign members to their respective codes. Raid groups usually consisted of 2-4 officers and 21 to 23 raiders (in total 25 members).

The tactical information scheme contains all audio fragments that were related to sharing information, making decisions and forming a strategy that was concerned with the content that was being done by the team during that session. In order to determine what code could be applied to an audio fragment several semantic rules were used.

Instruction: Sentences that implied that a different member had to perform an action, or perform an action in a certain way. "Do x", "watch out for y", "perform z 20 seconds into the fight". This code contains both overarching instructions, as well as direct warnings for certain occurrences in the game environment.

Negotiation: Any discussion about the current strategy that the team was using that involved one or more members. "Should we not do $\mathrm{x}$ in manner $\mathrm{z}$ instead of manner y", "No, manner $\mathrm{z}$ is superior to manner y because of factor a". This code contains suggestions, more in-depth discussions, and consensus reaching.

Reflection: Any audio fragments that were concerned with establishing what happened in the past. Whether it was about judging what went wrong for the team during the last attempt, or in order to establish what happened in a similar situation several days before.

Status: Any audio fragments that were concerned with players reporting to the group about their current condition, the state of the environment or the state of the group.

Action: Any audio fragments that were concerned with declarations of action and intention. "I'm going to do x".

\section{Results}

Two sessions were analyzed for both Team A and Team D to eliminate the chance of a fluke result. The Member scheme results (Fig. 1) indicate that Team A had several leaders that were equally active in their communication. The remaining 21 players that are coded under the Raider label formed the largest share of the communication over the course of the sessions. 35.9\% of all the audio recorded during the first session (Team A session 1, TAs1) was spoken by the regular raid members. During this session three of the four officers spoke almost an equal amount. Officer $124.0 \%$ of the time. Officers 2, 3 and 4 we're responsible for $17.9 \%, 19.3 \%$ and $3.0 \%$ of the audio respectively. It also seemed that the officers had a rough task distribution. Two officers (Officer1 and Officer3) were primarily concerned with explaining tactics, and one of these in particular initiated many reflective conversations after the team made an attempt at the boss they were trying to defeat. The second officer (Officer2) was primarily concerned with logistics, and made sure all team members were present, ready and had all their necessary avatar enhancements. Whenever an attempt was started he was always the person to give the go signal for the team to initiate the fight. The second session recorded for Team A (TAs2) was recorded during a slightly more 


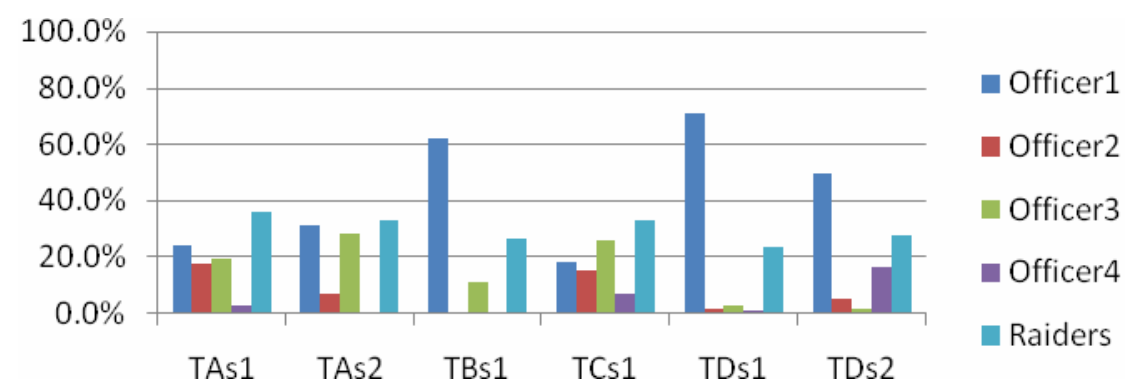

Fig. 1. Team member audio activity

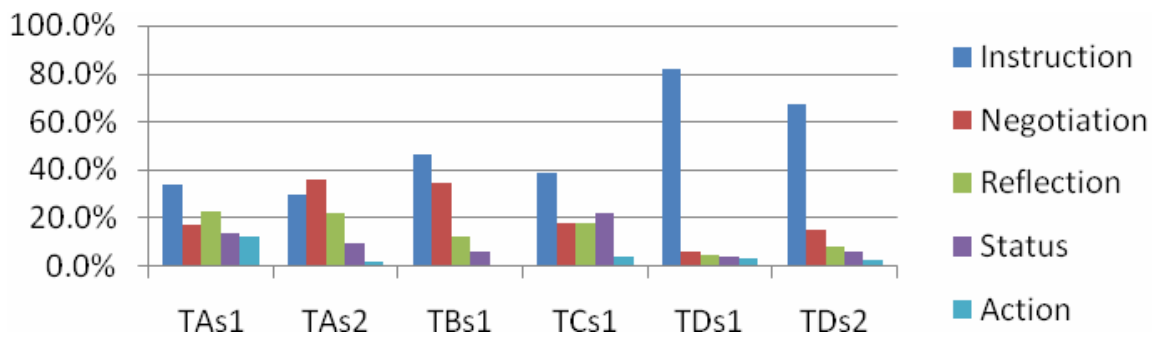

Fig. 2. Team communication type coverage. Notice the distribution among both of Team A's logs compared to both of team D's logs.

complex engagement. The team had quite some difficulties with the challenge and many attempts were made that usually ended fairly quickly. Officers 1 and 3 primarily concerned themselves with the tactics and spent a lot of time discussing among each other and with the rest of the team. Officers 1 and 2 were responsible for $31.5 \%$ and $28.5 \%$ of the audio respectively The second officer (6.8\%) was not in control of team logistics that session and was much less active in the discussions. The group of Raiders again formed the most active communication participant (33.3\%).

If we review Fig. 2 for Team A it is noticeable that all types of communication (instruction, negotiation, reflection, status and action) are all well represented in both sessions. Significant amounts of time were spent negotiating tactics, reaching team consensus and reflecting on past attempts. The first session contained $34.2 \%$ Instruction, $16.9 \%$ Negotiation, $23.1 \%$ Reflection, $13.6 \%$ Status and $12.1 \%$ Action messages. During Team A's second session negotiation was even the most populated code. The second session contained 30.1\% Instruction, 22.2\% Reflection, 36.3\% Negotiation, 9.4\% Status and $1.9 \%$ Action messages. In all other sessions, instruction was the most populated code. The problems encountered by Team A in this second session might explain their increased need to negotiate about a working tactic on the spot.

Team D had a very distinctly different communication pattern. Both of the sessions for Team D were recorded on the exact same fight as the first session for Team A (TAs1). Team D was controlled by a very directive officer (Officer1). On his own he tended to speak at least twice as much as the remaining 21 members of the group. The larger share of the group showed very passive behavior. The first session for Team D 
can be broken down as follows: Officer1 71.1\%, Officer2 1.4\%, Officer3 2.6\%, Officer4 $1.2 \%$, Raiders $23.7 \%$. Where Team A used four officers to handle the task workload of leading, Team D also had four present but only one of them was very communicative. The second session for Team D showed very similar results to the first session: Officer1 49.8\%, Officer2 4.9\%, Officer3 1.7\%, Officer4 $16.1 \%$, Raiders $27.5 \%$. This similarity is also found in the coded references of the tactical information scheme. The gap between Instruction and the other communication forms is very large in the data of Team D. The first officer was also the primary source for these Instructions. The tactical information scheme analysis for the first session contained $82.3 \%$ Instruction compared to $4.3 \%, 6.0 \%, 4.1 \%$ and $3.3 \%$ for Reflection, Negotiation, Status and Action respectively. The second session contained Instruction $67.4 \%$, Reflection $8.4 \%$, Negotiation $15.0 \%$, Status 6.4\%, and Action $2.9 \%$.

Let us compare a particular section of the data where distinct communication style differences between Team A and Team D occurred. We'll take a look at the same situation in logs TAs1 and TDs1, during which both teams were attempting to defeat the challenge 'Beasts of Northrend, Heroic'. During the first section of this fight the tanks ${ }^{1}$ had to rotate among them who was tanking the boss during the encounter based on what number of debuffs ${ }^{2}$ they had received from the boss. In Team A the tanks declared on their own how many debuffs they had, using a predetermined rotation order the tanks knew at what number of debuffs they had to take over and in which order they had to take over. These declarations of debuffs were coded as Status messages. Whenever a tank reached the number that was the trigger for a rotation to occur he would simply say the number over the VOIP channel and that would signal to the next tank in the rotation to take over. The tank that was taking over would often declare his action before taking over from the first tank; 'I'm about to take over' in order to inform the healers ${ }^{3}$ assigned to keeping the tanks alive to switch targets to the tank that was stepping in. These messages were coded as Action messages. This encounter was unforgiving enough that if a tank went without any healing for a period of 3 seconds he would very likely be dead. A death on the part of one of the tanks generally resulted in a failure for the entire team. This made the well coordinated switching of the tanks and healers very important.

In Team $\mathrm{D}$ this entire communication process was handled by the first officer. Whenever a tank reached the maximum amount of debuffs the officer would order one of other tanks to take over and would also instruct the healers to heal the tank that was stepping in. This put a lot of stress on the first officer and also led to create confusion among the tank rotation as well as the healers. Whenever the officer used a loud voice to instruct the healer to heal one of the tanks, healers with assignments other than healing the tanks would drop their original assignment and switch targets, which would then lead to increased risk to their original assignments. This deficient communication was the direct cause for several failed attempts and also created a very uncomfortable atmosphere in the group that caused the fight to be perceived to be harder than it actually was if the communication had been more distributed and

\footnotetext{
${ }^{1}$ Tanks: a particular role in the team filled by several members, their job is to protect the weaker members in the team by drawing the attention of threats to themselves.

${ }^{2}$ Debuffs: a temporary negative effect put on an avatar.

${ }^{3}$ Healers: players tasked with keeping other team members alive.
} 
calmer. The lack of reflection and negotiation during the sessions of Team D (Fig. 4.2) also indicated they failed to improve their tactics during the evening and were not able to determine what went wrong during attempts to make an improvement. Team A reached the $3^{\text {rd }}$ of this 3 phase fight near the end of the evening while Team D only managed to reach the $2^{\text {nd }}$ phase of the fight on their last attempt of the evening.

The data from Team A suggested that the ability to clearly define errors and problems were a critical part for their success. Defining where mistakes were made in the team and clearly declaring them made the team much more efficient and also created a more confident atmosphere. When the team wasn't succeeding they always knew why they were not succeeding. Among Team D this did not occur as much. Often the same set of instructions would get repeated even when no improvement was observed.

In order to verify if the found communication patterns can be extrapolated we analyzed the data of Team B ( 1300-1500) and C( 1500-1800) (both teams are positioned between Team A and D with respect to performance,. The session analysis for Team B resulted in Officer1 $62.0 \%$, Officer3 $11.3 \%$ and Raiders $26.7 \%$. Team B shared similarities with Team D in that they had a very authoritative leader. However, their tactical information scheme analysis shows a high amount of negotiation. The tactical analysis for Team B can be broken down as follows: Instruction $46.1 \%$, Reflection $12.4 \%$, Negotiation $34.6 \%$, Status $6.2 \%$ and Action $0.7 \%$. So, while Team B shared similarities with Team D's communication pattern, Team B was not nearly as instructional or authoritative. The session analysis for the slightly lower ranking Team C produced Officer1 18.5\%, Officer2 $15.4 \%$, Officer3 $26.1 \%$, Officer4 $6.9 \%$ and Raiders $33.2 \%$. They shared a similarly distribution of workload as Team A, but the number of references (almost twice as much references per time unit compared to Team A) coded for their session indicated that their communication was very fragmented and that members spoke often for very short lengths. This was reinforced by the results of the tactical information scheme. Team C's breakdown resulted in 38.5\% Instruction, 4.3\% Reflection, 6.0\% Negotiation, 22.2\% Status, $4.0 \%$ Action. Their number of Instruction references shared more similarities with Team D's tactical information scheme results.

\section{Discussion}

Very distinct differences were found between the communication patterns of the highest ranked team (position 1000), Team A, and the lowest ranked team (position 4000), Team D, in both of the sessions of each team. Large differences were evident in the member scheme (Fig 4.1) as well as in the tactical information scheme (Fig. 4.2). The higher ranked Team A showed a distributed communication pattern where communicative workload was equally divided between its leaders, and where the group of raiders, that numbered 21 members, was always the most vocal contributor to the total communication. The type of communication that was observed was also equally distributed; Team A showed high amounts of reflection and negotiation during their sessions. Because of the performance record of Team A we argue that this style of communication is an important asset of their team's success. 
The challenges these teams face in WoW can be described as a 25 entity collaborative reinforcement learning problem. Teams tend to spend dozens of attempts and many hours learning how to overcome a particular challenge while slight improvements are made with each attempt. We assess that it is very important for a team to have all its members learn and adjust to a new piece of content as quickly as possible. The team that can enable its members to learn fast ends up being successful, and we suspect that higher performing teams manage to achieve this through high interaction between all members of the team and very consciously negotiating and reflecting on the tactic they are developing to overcome the challenge they are facing. Interestingly enough, while the number of scheduled evenings per week and player level for Team $\mathrm{D}$ is roughly equal to Team A, their communication style was almost the exact opposite. Team D's communication was characterized by a single dominant leader who had the tendency to micromanage the team members actions. The remaining 24 members of the team were not nearly as communicative as this single leader and this is also well reflected in the communication type analysis.

Instructions were by far the most common, often coming from the dominant leader, but when other members of the team did speak up it was also often in the form of an instruction. The high amount of instructions coupled with a lack of reflection and negotiation generally meant the team tended to stick to their original plan and would not revise it very often after learning more about the content. This inflexibility and the low interaction between the various members led to a slow learning curve for the team. The results from Team B and C interpolated well between the results from A and D. In our study setup Team $\mathrm{C}$ was ranked lower than Team B, despite sharing slightly more similarities with Team A rather than Team D. However due to the way content was released and the time it took to record the necessary data the ranking difference between Team B and Team C is not as straightforward as it may seem. The guild ranking system is quite dynamic and new achievements by guilds quickly lower or raise a team's standing with about 100-300 points.

Our results replicate the results of other team related studies. In a closed experiment with novice users by Richter and Lechner, the most successful team was also the team using negotiation and reflective analysis the most [18]. However no data was available on the distribution of communication of each member of the 5 man team. A larger study of Change Management Teams also showed shared leadership as a more effective predictor of higher performing teams, however their research methods relied on two questionnaires applied at the start and end of a 6 month period[19].

Certain types of communication have different effects on how fast they contribute to the construction of a team's shared mental model [20]. Based on our results we suspect that negotiation and reflection contribute more effectively to shared mental model development than other communication types like instruction. The faster a team can switch from explicit communication to primarily implicit communication, when they are operating in an environment with a lot of time pressure, the faster it leads to a successful collaboration. Especially members of WoW raid groups benefit a lot from implicit communication as the act of communicating tends to negatively affect their ability to perform their in-game function during the team effort. 


\section{Conclusion}

Distributed leadership is a method of collaboration that involves multiple leaders that delegate and switch roles to each other and to non-leaders in order to facilitate the performance of the team. According to the results we gained from gathering data on four WoW teams in European top 1000 to 4000 , the better teams tend to use distributed leadership. The collaboration among the better teams in our study is marked by a high interaction between both leaders and followers and team strategy development with high levels of reflection and negotiation. The slightly lower ranked teams B and $\mathrm{C}$ use a somewhat distributed communication style. In contrast, the lower ranked team's collaboration (D) is characterized by a rigid command structure involving instruction and control of a dominant single leader. This suggests that the top teams in WoW are more likely to be using distributed leadership as opposed to a more topdown instructional approach that more commonly occurs in lower ranked teams. This means that there is a relation between the distributiveness of a team's communication pattern and the team's performance. As these processes are general aspects of team collaboration, and as other studies have found similar results in different environments (e.g. [19]), we argue that these findings can also be applied to other synchronous virtual teams that rely on a high information exchange in order to succeed, especially in areas like disaster relief and defense related team efforts. Communication in these teams is essentially information exchange. Learning which communication style is more prevalent in more successful teams can provide important knowledge on more effective information exchange $\&$ consensus reaching within virtual teams in general. Considering the novelty of the study method and the platform, it is an encouraging result for further team related studies in this area.

\section{References}

1. CHI Workshop on video games as research instruments, http://www.uclic.ucl.ac.uk/people/e.calvillo/ws_videogames/

2. Warner, N., Letsky, M., Cowen, M.: Cognitive model of team collaboration: Macrocognitive focus. Human Factors and Ergonomics Society Annual, USA (2005)

3. Broekens, J.: MMOGs as Social Experiments: the Case of Environmental Laws (2008) arXiv:0811.0709

4. Dannecker, A., Richter, S., Lechner, U., Dressner, N., Fabisch, S., Ilsemann, A.: Towards World of Warcraft as an experiment platform for teams. In: Americas Conference on Information Systems, USA (2008)

5. Martins, L.L., Gilson, L.L., Maynard, M.T.: Virtual Teams: What Do We Know and Where Do We Go From Here? Journal of Management, 805-835 (2004)

6. Kanawattanachai, P., Yoo, Y.: Dynamic nature of trust in virtual teams. Journal of Strategic Information Systems 2(2), 42-58 (2002)

7. de Lisser, E.: Update on small business: Firms with virtual environments appeal to workers. Wall Street Journal, B2 (1999)

8. Reeves, B., Malone, T., O’Driscoll, T.: Leadership's Online Labs. Harvard business review, USA (2008)

9. Jensen, J.A.: Joint Tactics, Techniques, and Procedures for Virtual Teams. USCINCPAC (2002) 
10. Rosen, M.A., Fiore, M.S., Salas, E., Letsky, M.: Tightly Coupling Cognition: Understanding How Communication and Awareness Drive Coordination in Teams. The international C2 Journal, USA 2(1) (2008)

11. Salas, E., Cooke, J., Rosen, M.A.: On Teams, Teamwork, and Team Performance: Discoveries and Developments. Human Factors 50(3), 540-547 (2008)

12. Powell, A., Piccoli, G., Ives, B.: Virtual Teams: A Review of Current Literature and Directions for Future Research. The DATA BASE for Advances in Information Systems, USA 35(1) (2004)

13. Reeves, B., Malone, T.: Leadership in Games and at Work: Implications for the Enterprise of Massively Multiplayer Online Role-Playing Games. Seriosity, Inc., Palo Alto (2007)

14. Cohen, S.G., Bailey, D.E.: What makes teams work: Group effectiveness research from the shop floor to the executive suite. Journal of management 23(3), 239-290 (1997)

15. Alavi, M., Yoo, Y.: Is Learning in Virtual Teams Real? Harvard, Boston (1997)

16. DeSanctis, G., Poole, M.S.: Transitions in teamwork in new organizational forms. Advances in group processes 14, 157-176 (1997)

17. Jarvenpaa, S.L., Leinder, D.E.: Communication and trust in global virtual teams. Organization science 10(6), 791-815 (1999)

18. Richter, S., Lechner, U.: Transactive Memory Systems and Shared Situation Awareness: a World of Warcraft experiment. In: International Conference on Organizational Learning, Knowledge and Capabilities, The Netherlands (2009)

19. Pearce, C.L., Sims Jr., H.P.: Vertical Versus Shared Leadership as Predictors of the Effectiveness of Change Management Teams: An Examination of Aversive, Directive, Transactional, Transformational, and Empowering Leader Behaviors. Group Dynamics: Theory, Research, and Practice 6(2) 172-197, (2002)

20. Mathieu, J.E., Heffner, T.S., Goodwin, G.F., Cannon-Bowers, J.A., Salas, E.: The Influence of Shared Mental Models on Team Process and Performance. Journal of Applied Psychology 85(2), 273-283 (2000) 